\title{
Nephroprotective Effect of a Unani drug Mako (Solanum nigrum) in Gentamicin induced Nephrotoxicity in Rat Model
}

\author{
Mohd Aslam ${ }^{1}$, Shabana Rehmen ${ }^{2}$, Kalim Javed $^{3}$, Shahid Chaudhary ${ }^{2}$ \\ ${ }^{1}$ ILMUL ADVIA (UNANI PHARMACOLOGY), JAMIA HAMDARD, India, ${ }^{2}$ Dept. of Ilmul Advia, School of Unani \\ Medicine, Jamia Hamdard, India, ${ }^{3}$ Dept. of Chemistry, School of Science, Jamia Hamdard, India
}

\section{Abstract}

Background: Chronic kidney disease (CKD) is an increasingly common condition worldwide. According to the 2010 Global Burden of Disease study that ranked the causes of death worldwide in 1990 and 2010, CKD climbed to the 18th position over two decades. The prevalence of CKD in India varies from $0.16-0.78 \%$. A large number of chemicals in common use are potential renal toxins. Use of natural compounds for nephroprotective effect is on rise because of their low toxicity and comparatively higher safety. Solanum nigrum has been used in Unani system of medicine since time antiquity in ascitis, piles, fever, gonorrhea, liver diseases and renal diseases.

Methods: In this study, methanolic extract of Solanum nigrum (ME) was studied for its nephroprotective activity against gentamicin $(100 \mathrm{mg} / \mathrm{kg})$, induced acute renal injury in Wistar rats of either sex. In the experimental regimen, the animals were administered with ME (p.o.) at dose levels of $200-800 \mathrm{mg} / \mathrm{kg}$ (equivalent to $5-20 \mathrm{gm}$ of the traditional therapeutic crude dose respectively), for 10 days. Gentamicin (100 mg/kg, i.p.) was administered in a single dose from 4th day to 8th day of the experiment.

Results: The results showed significant $(\mathrm{p}<0.01)$ reduction in the elevated blood urea, serum creatinine, uric acid and also normalized the histopathological changes. However, the results were comparatively better at $800 \mathrm{mg} / \mathrm{kg}$ dose level.

Conclusion: The findings suggest that the ME possesses marked nephroprotective activity with minimal toxicity and could offer a promising role in the treatment of acute renal injury caused by nephrotoxins like gentamicin. It is a potential plant can be used as nephroprotective agent. 